\title{
Determination of the Concentration of Polyamines with SPR-based Immune Biosensor for Early Diagnostics of Breast Cancer
}

Maksym $\mathrm{P}^{1}$, Starodub $\mathrm{N}^{2 *}$ and Bilko $\mathrm{N}^{3}$

${ }^{1}$ Department of Laboratory Diagnostics of Biological Systems, National University of Kyiv-Mohyla Academy, Kyiv, Ukraine

${ }^{2}$ Department of Microbiology and Biotechnology, National University of Environmental Sciences, Kyiv, Ukraine

${ }^{3}$ Department of Laboratory Diagnostics of Biological Systems, National University of Kyiv-Mohyla Academy, Kyiv, Ukraine

\begin{abstract}
The paper presents the results of research on the development of immune biosensor test system for express detection of polyamines in cells of breast cancer. Determination of polyamines was performed by using an analytical device - immune biosensor based on surface plasmon resonance (SPR), where "antigen-antibody" reaction is performed in real time on the surface of transducer, resulting in formation of immune complexes and recording the shift of resonance angle. The method can detect the studied polyamines spermine and spermidine in concentration less than $10 \mathrm{ng}$ in $1 \mathrm{~mL}$, and with increasing concentrations a statistical probability of the analysis' result rise sharply. Moreover, the dependence of sensitivity of biosensor response from the concentration of polyamines is in the range of $10-100 \mathrm{ng} / \mathrm{mL}$.
\end{abstract}

Keywords: Polyamines; Antibody; Antigen; Surface plasmon resonance; Breast cancer

\section{Introduction}

The most common among the all types of cancer it is breast one and it is especially common in elderly aged women. This makes the urgency of developing new and improving existing methods of diagnosis of this disease. The extreme complexity of carcinogenesis and the difficulty of early detection of the breast cancer are aiming paramount importance - the choice of optimal treatment option [1]. Breast cancer - systemic disease that is primarily associated with dysfunction of the human immune system. The growth of the tumor, usually caused by systemic disorders of cellular and humoral immunity with a prevalence of such processes as the activation of suppressor cells, increased inhibitory processes in the blood, inhibiting the endocrine function of thymus, increased glucocorticoid function of adrenal cortex [2]. Over the last 10 years, researchers in the field of immunology and immunotherapy have made significant progress in the study of breast cancer. So for the diagnosis of various cancers commonly used methods are based on detection of biomarkers - molecules specific to certain types of cells. Today, such methods are widespread, in particular, the enzyme-linked immune sorbent assay (ELISA), where specific antibodies and enzyme tags are used for detection, real-time polymerase chain reaction (RT-PCR) to identify the nucleotide sequences of genes that code a particular disease specific protein. However, today there is necessary to have much simpler cheaper and more accurate methods for analysis than mentioned above. Quite promising example of such methods it is use of immune biosensors that can detect specific molecules in very low concentrations using specific antibodies to specific markers for certain types of cancer. Development of techniques with use of biosensors will greatly facilitate the diagnosis of different types of cancer, especially in the early stages of the disease. For early diagnostics, evaluation of the effectiveness of treatment of malignant tumors, metastasis detection, the determination of substances, the level of which can be associated with the presence of cancer is being performed. These substances are called tumor markers.

\section{Materials and Methods}

\section{Materials}

The materials for the research were highly specific serums (ab 7318 and ab 26975), obtained by immunizing rabbits that were provided by Abcam (Cambrige, England) against the polyamines of spermine and spermidine (Sigma-Aldrich), which were provided by the Kavetskyi Institute of Experimental Pathology, Oncology and Radiobiology and analytical instrument immunobiosensor "Plazmontest", which is an optical device based on the SPR, equipped with a sensor with resolution 2048 pixels, which is connected directly to the computer and registers and processes the received optical signal. The device was developed at the Glushkov Institute of Cybernetics of NAS of Ukraine.

\section{Immobilization procedure}

With the help of imunobiosensor we registered antigen-antibody interactions in real time. The feature of immunosensor "Plazmontest" is that the sensitive layer is formed on a glass plate, the surface is covered with adhesive $(1-2 \mathrm{~nm}$ ) layer of niobium and $50 \mathrm{~nm}$ gold film that provides the appearance of SPR. During the fall of plane-laser beam on the surface of the gold layer, in certain (critical) angle, appears the phenomenon of SPR - oscillations of the charges density on the edge of two media with metal and dielectric. Part of the beam energy is spent on oscillation and thus the intensity of the reflected beam at certain (critical) angle decreases, and the reflected angle becomes a constant characteristic of the particular condition of transducer. During immobilization of antibodies on the surface of gold, the critical angle varies, and the magnitude of the angle shift is directly dependent on the concentration of reagent which is being determined. Usually the determination sensitivity of a number of biological analytes is at the level of $5 \mathrm{ng} / \mathrm{mL}$.

*Corresponding author: Nikolay Starodub, Department of Microbiology and Biotechnology, National University of Environmental Sciences, Kyiv, Ukraine, Tel: +38044527824; E-mail: nikstarodub@yahoo.com

Received November 16, 2016; Accepted November 24, 2016; Published December 04, 2016

Citation: Maksym P, Starodub N, Bilko N (2016) Determination of the Concentration of Polyamines with SPR-based Immune Biosensor for Early Diagnostics of Breast Cancer. J Biosens Bioelectron 7: 227. doi: 10.4172/2155-6210.1000227

Copyright: @ 2016 Maksym P, et al. This is an open-access article distributed under the terms of the Creative Commons Attribution License, which permits unrestricted use, distribution, and reproduction in any medium, provided the original author and source are credited. 
When exceeding the critical angle of plane-polarized light beam, in case of highest values of refraction, the total internal reflection occurs. Under these conditions, it is observed resonance minimum of the dependance of intensity of emmition refraction from the reflection angle of the laser beam on the film of gold due to overloading of emmiting energy. The interaction of antigen specific antibody to specific immunogen (in our case polyamines) is being recorded by changing the angle of reflection angle similarly to dependence mentioned above, which makes possible monitoring of antigen binding to the antibody and eventually cause the high sensitivity in determining the level of antigen, and hence the opportunity early diagnosis and statistically significant polyamine concentration in control solutions and in cells. According to recent literature data, immobilization of antibodies on the surface of pure gold is quite inefficient to recognize antigens as specific binding sites are blocked, because the antibodies bind to the surface randomly. Therefore it is advisable to carry out preliminary preparation of transducer by application of various substances which provide site-oriented binding of antibodies. In such cases, the activity of the antibodies may increase to $>70 \%[3,4]$.

\section{General procedure analysis with biosensor}

So, in our case, the preparation of the working surface of biosensor was performed in such way shown in Figure 1. Firstly we covered the golden surface of the transducer with polyelectrolyte insoluble film using polyallilamine hydrochloride (PAA) at a concentration of $1 \mathrm{mg} /$ $\mathrm{mL}$, then applied a solution of protein A from Staphylococcus aureus in concentration of $1 \mathrm{mg} / \mathrm{mL}$. After applying the surface of transducer with protein A we managed to absorb polyclonal antibodies specific to spermine or spermidine, then applied bovine serum albumin (BSA) to block the free areas on the gold surface, at a concentration of $1 \mathrm{mg} /$ $\mathrm{mL}$. As a rule, application of BSA did not change significantly the value of the resonant angle, what means that on the sensitive surface of biosensor actually didn't left empty places for the binding and antibody concentration was sufficient to create the most dense layer. The next stage of the experiment was applying solutions of different concentrations of polyamines spermine and spermidine. From the stock solution we prepared 6 working solutions of different concentrations of polyamines from 10 to $100 \mathrm{ng} / \mathrm{mL}$. Exposure time of each solution was $10 \mathrm{~min}$ at $25^{\circ} \mathrm{C}$, whereas further change in resonant angle was observed. At each stage, the cells were washed with saline solution. After that the calibration curves were created and analyzed (Figure 1).

\section{Direct algorithm of analysis}

Since both analyzed polyamines immunologically are haptens, among different algorithms of analysis, it was used three, which are the following: "direct", when the immobilized specific Ab directly interacting with analyte which is shown on Figure 2. In this method of immobilization we directly immobilized specific antibodies on transducer surface, only with help of physical absorption and after modification of surface with PAA, protein $\mathrm{A}$ and further addition of BSA we started to measure a resonant angle after exposure of polyamines (Figure 2).

\section{Competitive algorithm of analysis}

During the competitive algorithm we conjugated studied polyamines with BSA (with help of glutaraldehyde solution) immobilized on the transducer surface. Then we added complex mixture of free polyamines and antibodies to such specific layer together (Figure 3).
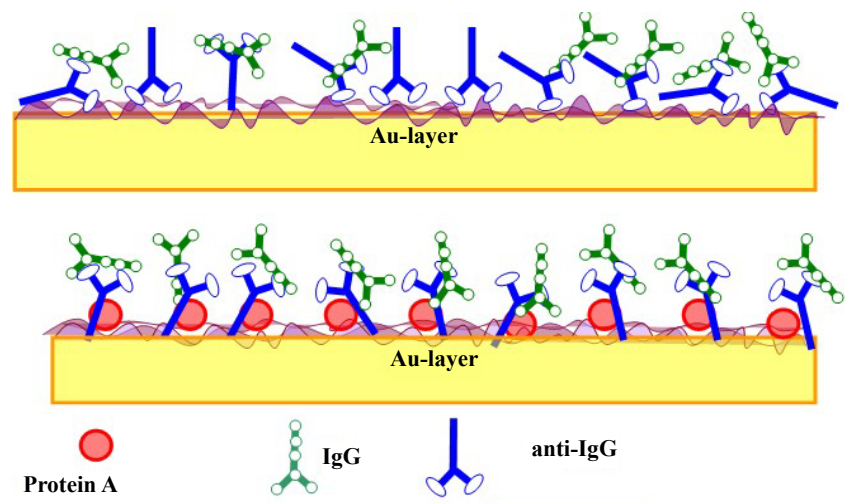

MuMbuhuming Polyelectrolite

Figure 1: Preparation of transducer surface and modification.

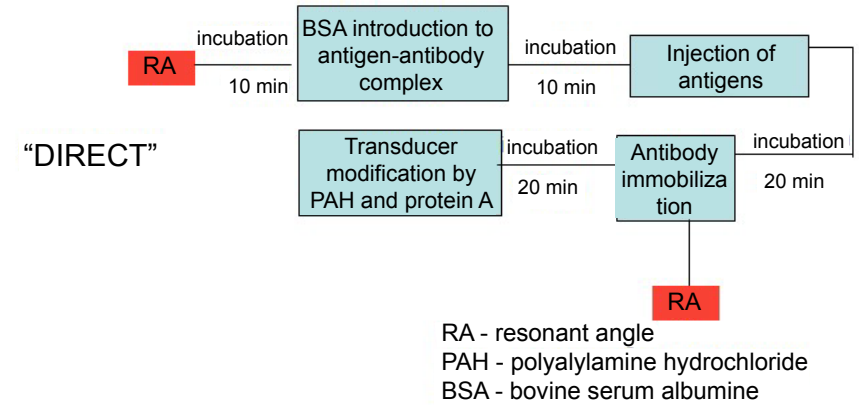

Figure 2: Direct algorithm of polyamine analysis.

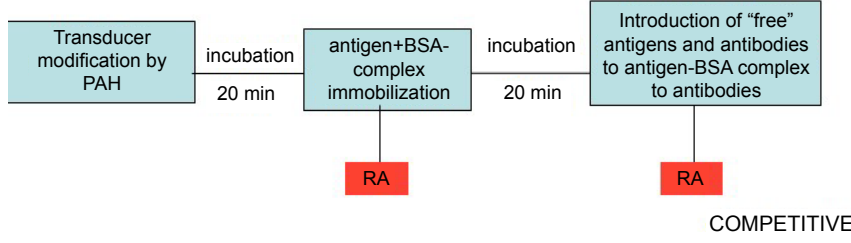

Figure 3: Competitive algorithm of analysis.

\section{Results and Discussion}

\section{Determination of the biosensor sensitivity}

Firstly, we determined the sensitivity of our biosensor by making various concentration of polyamine solutions from 10 to $100 \mathrm{nM} / \mathrm{mL}$. Also we used various algorithms of analysis in order to optimise the procedure of analysis. According to the data obtained we could state that range of sensitivity for our biosensor for spermine and spermidine is from $10 \mathrm{ng} / \mathrm{mL}$ to $100 \mathrm{ng} / \mathrm{mL}$ during direct algorithm of analysis and the lowest concentration on which we could see clear response is about 10 ng. Also we could see better results during competitive analysis because because the lowest concentration on which we could detect the clear signal of change of resonant angle is $0,3 \mathrm{ng} / \mathrm{mL}$ in spermine and 0,05 $\mathrm{ng} / \mathrm{mL}$ in spermidine. So we also could see better detection sensitivity during analysis of spermidine because the lowest concentration on which we could detect this polyamine. Furthermore as we could see the range of detection is also more wider during competitive analysis 
and again we could see better results of detection of concentration of spermidine. So as we could see our method of detection of polyamine concentration is quite accurate. Also this method of analysis could be quite fast if make the modification of the surface of transducer before the analysis. In these conditions all procedure of analysis could be performed up to $20 \mathrm{~min}$. Our method has only one significant problem that we encountered during experiment. This problem in that there is a $20 \%$ possibility of cross reaction between spermidine and spermine which could case difficulties in further research of biological fluids such as blood plasma and urine. Nevertheless we are trying to solve this problem what should be describes in further research. All of our results are presented on Table 1 (Figures 4 and 5 and Table 1).

\section{Theoretical study}

Experience in the use of tests for detection of tumour markers showed that their introduction into medical practice significantly increases efficiency of the diagnostics. Cancer markers are products of metabolism of neoplastic cells, which can be quite specific to one type of tumour and show the presence of cancer. In healthy people, these compounds are usually absent or determined in small quantities. Abnormal expression of the genome that occurs in tumour cells leads to violations of such basic processes as differentiation and proliferation. In these cells metabolic processes are changing, which is accompanied by production of certain compounds. From the compounds that are produced by normal cells, they differ qualitatively or quantitatively. All markers are divided into: markers that are produced and secreted in biological fluids only in tumour cells (tumour markers); markers, production and secretion of which is the result of metabolic disorders during cancer. They can be specific and non-specific (tumourassociated markers); markers that appear in the case of hormonedependent tumours found in cytoplasm membrane of tumour cells and perform receptor function. The level of tumour markers depends on many factors. In particular, the properties of the tumour, the treatment and biochemical feature of the marker. In patients with non-cancer pathology level of tumour markers typically does not exceed the normal range. However, it is needed to consider the possibility of non-specific increase of their level. In particular, it may increase during pathological processes such as inflammation of liver, pancreas, lungs and so on. In

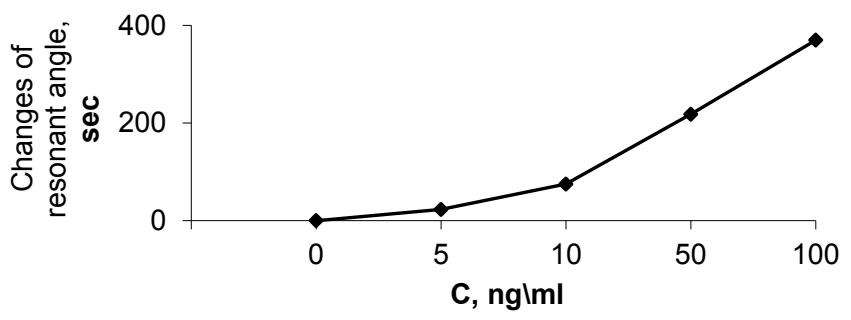

Figure 4: Direct algorithm of analysis, calibration curve.
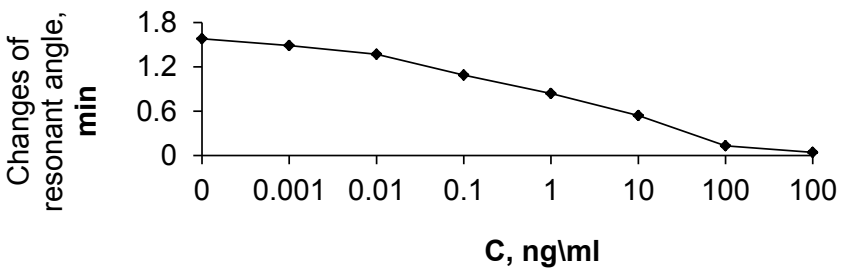

Figure 5: Competitive algorithm of analysis, calibration curve.

\begin{tabular}{|c|c|c|}
\hline $\begin{array}{l}\text { Indexes and algorithm of } \\
\text { analysis }\end{array}$ & $\begin{array}{c}\text { Sensitivity of determination, } \\
n \text { glmL }\end{array}$ & $\begin{array}{l}\text { Linear region, } \\
\text { nglmL }\end{array}$ \\
\hline \multicolumn{3}{|c|}{ Spermine } \\
\hline direct & 10 & $10-100$ \\
\hline competitive & 0,3 & $0,3-80$ \\
\hline \multicolumn{3}{|c|}{ Spermidine } \\
\hline direct & 10 & $\leq 10-100$ \\
\hline competitive & 0,05 & $0,01-90$ \\
\hline
\end{tabular}

Table 1: Comparison of determination sensitivity of spermine and spermidine.

modern oncology practice, research of tumour is markers conducted in order to perform cancer pathology screening, assessment of prevalence of disease (a subsidiary criterion), diagnostics of recurrences and metastases and evaluating the effectiveness of treatment.

Structure and features of polyamines: Polyamines associated with different biological processes [5]. These active biogenic amines involved in a number of basic cellular functions, including cell growth, proliferation and differentiation, and cellular mechanisms regulating the stress response and cell survival. Spermidine and spermine have anti-inflammatory and antioxidant properties [6]. And thus were involved in cellular and organism protection in aging and disease processes [7]. Thus it is shown that spermidine increases life time. On the other hand, polyamines can also be toxic to cells at high levels and may contribute to cell death, mainly via oxidative mechanisms $[8,9]$. In this regard, polyamines are important clinical and biochemical markers for malignant tumours. For the first time polyamines were seen in the urine of cancer patients, where they were present at a higher level in cancer patients than in healthy patients [10].

According to the research data the basic level of biogenic polyamines in healthy people is at nanomolar concentrations (average $45 \mathrm{nM}$ ). So spermine concentration in the blood of healthy men is $5,40 \pm 1,23 \mathrm{nM} /$ $\mathrm{mL}$ in women $7,07 \pm 1,03 \mathrm{nM} / \mathrm{mL}$, and spermidine - 7,54 $\pm 1,32 \mathrm{nM} /$ $\mathrm{mL}$ in men and 9,95 $\pm 1,11 \mathrm{nM} / \mathrm{mL}$. Also, according to the recent data there was performed a research for determination of concentration of polyamines in saliva [11]. So healthy spermine and spermine levels in saliva is $0.5 \mathrm{nM} / \mathrm{mL}$, and can also be used as a diagnostic indicator [12]. So we could say that OU biosensor could determine the concentrations of polyamines approximate to normal values.

Polyamines as tumour markers: Most types of breast cancer is easily diagnosed using microscopic analysis of biopsy. While screening methods are useful in the early diagnosis of cancer, further testing is necessary to determine the status of the disease. Today a number of screening tests are applied, including clinical tests and mammography. However, women do not want to undergo testing because of shyness, spending time and high cost. Thus, the search for a new simple, convenient and cheap way of screening is very necessary. There are many different tumour markers that indicate a particular disease process, and they are used in oncology to help detect the presence of cancer. The concentration of tumour markers, such as carcinoembryonic antigen (CEA) and cancer antigen CA15-3, significantly increased in patients with breast cancer. However, elevated levels of tumor markers can also be caused by other reasons. These markers are macromolecular compounds such as enzymes and glycoproteins. Polyamines which are low molecular weight compounds, could be associated with rapid tumor growth through their biosynthesis and accumulation [13]. Thus, the concentration of polyamines such as spermine and spermidine, increasing in blood plasma and urine [14-16]. Therefore, polyamines, could be an important biochemical tumor markers. 
Immunoassay of polyamines: The following immunoassay methods that have been used for the determination of polyamines are: radioimmunoassay (RIA) and ELISA. Both methods provide high sensitivity require small tissue samples and can analyze multiple samples in parallel. Moreover, there is no need derivatization of samples. However, the main problem is the specificity of antibodies and danger of radioactive reagents using RIA. The first efforts to the production of antibodies to spermine led to the synthesis of antibodies that have low specificity towards spermine and had a high rate of cross reaction with spermidine [17]. ELISA, in which the antibodies against both spermine and spermidine were used, was inaccurate again because of low specificity of antibodies [18]. The disadvantages of both methods are: necessity a long time for analysis and impossible its fulfilment in field conditions.

\section{Conclusions}

According to the obtained results we made further conclusions:

- The sensitivity of the determination of spermine and spermidine by the "direct" algorithm of analysis is on the level of $10-100 \mu \mathrm{g} / \mathrm{mL}$

- Application of competitive algorithm of analysis with simultaneous including of the intermediate layer from PAA promoted further increase of the determination level of both polyamines.

- Competitive algorithm of analysis shown some sensitivity increase of both polyamines determination in the linear range of determination.

- The comparison of the sensitivity of the immune biosensor analysis of the both types of polyamines with was given by ELISA method in the literature gives possibility concluding that the developed novel approach could be able to provide control of polyamines according to the practice demands in appropriate sensitivity rate. Since the overall time of biosensor analysis is about $30-40 \mathrm{~min}$ and it could be shortened up to $10-20 \mathrm{~min}$ if the transducer surface will be preliminary prepared. The transducer surface and its preliminary treatment by the above mentioned chemicals (PAA, protein A), may be fulfilled by so called integral way as it was realized in electronic technology. It makes this method of analysis much cheaper than ELISA and even less, since some labelled components are excluded.

\section{- Our tasks for further research}

The next our investigations will be directed on the solving such questions:

1) At first, revealing specificity and sensitivity of the both polyamines determining such biological fluids as the serum blood and urine;

2) Next, searching optimal ways of the extraction of polyamines from some types of tissues for the quantitative determination of these analytes with the help of the immune biosensor;

3) At last, comparing efficiency of determination of the above mentioned polyamines (in such tissues and body fluids as blood serum, urine and tumour tissue) with the number of traditional approaches used on the practice.

\section{References}

1. Mayevkyi OY (2014) Breast cancer. Modern Methods of Diagnosis Using Oncomarkers, Specific Immunotherapy. Journal of Vinnista 18: 635-640.
2. Osynskyi SP (2010) Metabolic environment of cancer cells. Oncology pp: 197-218.

3. Qi C, Gao G, Jin G (2011) Label-free Biosensors for Health Applications. Pier Andrea Serra (ed), Biosensors for Health, Environment and Biosecurity, InTech pp: 550 .

4. Starodub NF (2013) Efficiency of Biosensors in Environmental Monitoring Jones BE, Huang $\mathrm{H}$ (eds), Portable Biosensing of Food Toxicants and Environmental Pollutants. Series in Sensors, London, NewYork pp: 515-560.

5. Gugliucci A (2004) Polyamines as clinical laboratory tools. Clinica Chimica Acta 344: 23-35.

6. Zhang MH, Caragine T, Wang HC, Cohen PS, Botchkina G, et al. (1997) Spermine inhibits proinflammatory cytokine synthesis in human mononuclear cells: a counter regulatory mechanism that restrains the immune response. $J$ Exp Med 185: 1759-1768.

7. Minois N, Carmona-Gutierrez D, Aging FM (2011) Polyamines in aging and disease. Aging (Albany NY) 3: 716-732.

8. Brunton VG, Grant MH, Wallace HM (1990) Spermine toxicity and glutathione depletion in BHK-21/C13 cells. Biochem Pharmacol 40: 1893-1900.

9. Morgan DML (1990) Polyamines and cellular regulation: perspectives. Biochem Soc Trans 18: 1080-1084.

10. Russell DH (1971) Increased polyamine concentrations in the urine of human cancer patients. Nat New Biol 233: 144-145.

11. Takayama T, Tsutsui H, Shimizu I, Toyama T, Yoshimoto N, et al. (2016) Diagnostic approach to breast cancer patients based on target metabolomics in saliva by liquid chromatography with tandem mass spectrometry. Clinica Chimica Acta 452: 18-26

12. Voronchihina LD, Demyanova VT, Sitnikov SA (1986) Polyamine concentration in the blood of healthy people. Problems of Medical Chemistry 22: 43-45.

13. Russell D, Snyder SH (1968) Amine synthesis in rapidly growing tissues: ornithine decarboxylase activity in regenerating rat liver, chick embryo, and various tumors. Proc Natl Acad Sci 60: 1420-1427.

14. Suh JW, Lee SH, Chung BC, Park J (1997) Urinary polyamine evaluation for effective diagnosis of various cancers. J Chromatogr B 688: 179-186.

15. Lee SH, Kim SO, Lee H, Chung BC (1998) Estrogens and polyamines in breas cancer:their profiles and values in disease staging. Cancer Lett 133: 47-56.

16. Hiramatsu K, Miura H, Kamei S, Iwasaki K, Kawakita M (1998) Development of a sensitive and accurate enzyme-linked immunosorbent assay (ELISA) system that can replace HPLC analysis for the determination of $\mathrm{N} 1, \mathrm{~N} 12$ diacetylspermine in human urine. J Biochem 124: 231-236.

17. Bartos D, Campbell RA, Bartos F, Grettie DP (1975) Direct determination of polyamines in human serum by radioimmunoassay. J Cancer Res 35: 2056-2060.

18. Fujiwara K, Asada H, Kitagawa T, Yamamoto K, Ito T, et al. (1983) Preparation ofpolyamine antibody and its use in enzyme immunoassay of spermine and spermidine with `D-galactosidase as a label. J Immunol Methods 61: 217-226. 